\title{
INTERSTITIAL KERATITIS
}

Interstitial keratitis denotes any inflammation affecting the corneal stroma. In this context its use will be limited to a stromal keratitis which is diffuse, disciform, or irregular in distribution, and will exclude that usually described as punctate.

\section{Physical Signs}

The following changes are seen with the slit-lamp microscope:

(1) Cloudiness of the corneal stroma varying from a reversible milky opalescence to permanent scarring.

(2) Vascularization of the opacity, which is an inconstant feature. Classically the vessels are deeply situated, arising from the anterior ciliary arteries, but the superficial circumcorneal plexus often contributes to the vascularization.

(3) Increase of corneal thickness during the stage of active keratitis resulting from the separation of stromal lamellae by oedema and inflammatory cells, with a reversion to normal when the condition is quiescent, or to facet formation and irregular thinning.

\section{Pathogenesis}

Two processes contribute towards the production of the inflammatory changes:

(1) Direct infection. The responsible organisms invade the stroma from the corneal epithelium, uveal tract, limbal circulation, or an adjacent scleral focus.

(2) Antigen-antibody interaction. This is a particular feature of the keratitis resulting from viral or treponemal infections. The most peripheral site of interaction of antigen and antibody may present as a ring concentric with the limbus-a characteristic feature of viral keratitis (Fig. 1).

\section{Related Systemic Manifestations}

Interstitial keratitis may exist alone or be but one aspect of widespread systemic disease. Recognition of these systemic features may elucidate the aetiology of the keratitis.

\section{(1) Interstitial Keratitis with Skin Lesions}

(a) Local Vesicular Eruptions.-Herpes simplex is the most common cause of the disciform type of stromal keratitis. A history of cold sores is usual but not inevitable. The condition is characterized by repeated attacks of keratitis which are usually central but which may be eccentric or diffuse. The duration of activity is extremely variable. Recurrences may be triggered off by fever, sunbathing, or minor trauma. Dendritic ulceration sometimes precedes or complicates the condition.

Herpes zoster ophthalmicus occasionally gives rise to a disciform keratitis although coarse punctate opacities are more commonly found. The severe vesiculo-bullous skin eruption is characteristic, and when the lateral side of the nose is affected, the cornea is usually involved.

(b) Exanthemata.-Varicella, variola, and vaccinia are characterized by their widespread vesicular eruptions, although vaccinia may involve the conjunctiva and eyelids as a local manifestation of auto-inoculation. Primary corneal infection may occur, but secondary involvement from the lids is more common.

(c) Miscellaneous Skin Conditions.-Onchocerciasis manifests itself by the presence of subcutaneous nodules harbouring the adult worm. Early corneal involvement, resulting from the death of intra-corneal microfilariae, is characterized by a coarse punctate keratitis, but a severe stromal keratitis may result if the condition is left untreated. 


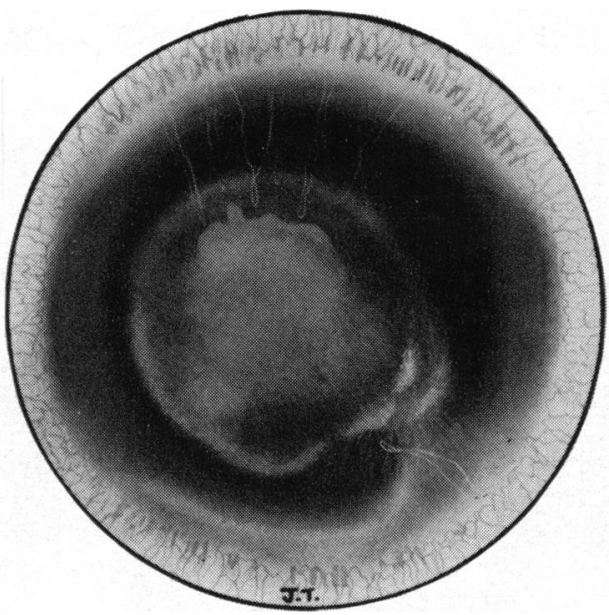

FIG. 1.-Vaccinial interstitial keratitis.

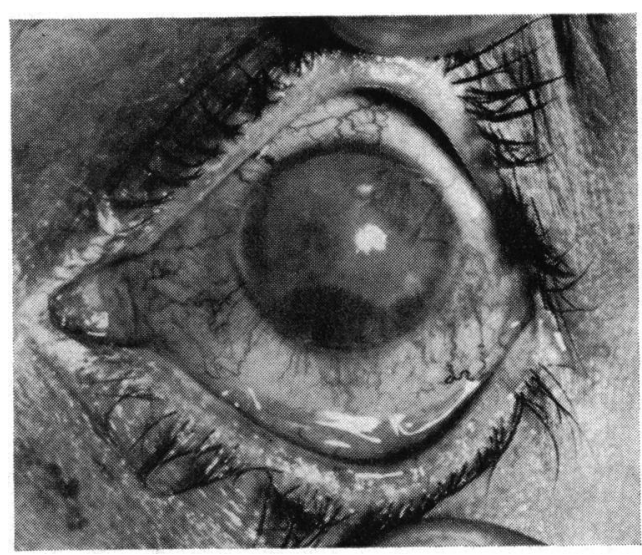

FIG. 2.-Syphilitic interstitial keratitis.

Leishmaniasis can produce an interstitial keratitis, probably the result of direct transfer of organisms from a local sore.

Hodgkin's disease, mycosis fungoides, and Kaposi's sarcoma are all rarely associated with a stromal keratitis, the pathogenesis of which is ill understood.

\section{(2) Interstitial Keratitis with Auditory Symptoms}

Congenital syphilis is the classic but now infrequent cause of interstitial keratitis. It usually presents with characteristic ghost vessels leading to deep irregular stromal opacities in an otherwise quiet eye. Typical salmon patches and vascularization en épaulette are rarely seen today (Fig. 2). Over half the cases are bilateral and are frequently associated with a disseminated chorioretinitis. Keratitis, nerve deafness, and pegged incisor teeth constitute Hutchinson's triad.

Cogan's syndrome is characterized by recurrent attacks of interstitial keratitis and nerve deafness which sometimes follow vaccination or drug therapy. Tinnitus and vertigo are prominent symptoms. This syndrome may be a manifestion of auto-immune disease.

\section{(3) Interstitial Keratitis with Glandular Enlargement}

Mumps is rarely complicated by a stromal keratitis which, although severe, is transient.

Lymphogranuloma venereum can produce a localized irregular stromal keratitis. The primary infection is usually genital, associated with inguinal buboes, but rarely it is conjunctival.

Tuberculosis, syphilis, sarcoidosis, and Hodgkin's disease may all present with keratitis and lymphadenopathy. In tuberculosis the keratitis is particularly localized to the lower part of the cornea and concomitant scleritis and uveitis are common. In sarcoidosis the stromal keratitis may result from direct corneal involvement or may be related to an anterior uveitis.

\section{Treatment}

In general this involves the eradication of the cause by local and systemic therapy. Local antibacterial and antiviral agents can be used to counter any direct infection. If the stromal disease is principally an immunological reaction, local and systemic steroids may be used cautiously. Therapeutic and optical keratoplasty afford a means of removing diseased and scarred corneal tissue.

\section{Illustrations:}

INSTITUTE OF OPHTHALMOLOGY, LONDON, W.C.1.

\section{A. Patterson,} THE LONDON Hospital, LoNDON, E.1. 